\title{
Does the type of receptacle influence the crossmodal association between colour and flavour? A cross-cultural comparison
}

\author{
Xiaoang Wan ${ }^{1,2^{*}}$, Carlos Velasco ${ }^{2}$, Charles Michel ${ }^{2}$, Bingbing $\mathrm{Mu}^{1}$, Andy T Woods ${ }^{3}$ and Charles Spence ${ }^{2}$
}

\begin{abstract}
Background: We report a cross-cultural study designed to investigate whether the type of receptacle in which a coloured beverage is presented influences the colour-flavour associations that consumers make. Participants from the United States of America (USA) and China were shown photographs of red, green, yellow, blue, orange, brown, and clear liquids in a water glass, a wine glass, a cocktail glass, and a plastic cup.

Results: The two groups of participants exhibited different colour-flavour associations for the green, yellow, orange, and brown drinks when these were presented in the different receptacles, suggesting some interesting interactions between the receptacle, colour, and flavour. Cross-cultural differences were also observed in the colour-flavour associations for red and blue drinks that were independent of the type of container in which the drinks were presented.

Conclusions: These findings highlight the existence of an interaction between contextual factors (the receptacle in which a drink is presented) and the cultural background of our participants (China versus the USA) in terms of colour-flavour associations. Such results raise interesting questions regarding the underlying mechanisms responsible for these effects.
\end{abstract}

Keywords: Colour-flavour interactions, Tableware, Cross-cultural difference, Contextual factors

\section{Background}

Perceiving the flavour of foods and drinks involves a process of multisensory integration [1,2]. The colour of a food or drink item may, for example, provide clues regarding the likely identity and intensity of the flavour $[3,4]$. Yet, flavour perception is also influenced by cognitive factors such as a person's expectations, which may lead people to generate false expectations and to misidentify flavours and/or odours [5-11].

Colours convey different aesthetic values and meanings in different contexts [12], so it would seem likely that consumers from different cultures might have different expectations when presented with the same coloured beverage. Evidence in support of this suggestion comes from Shankar,

\footnotetext{
*Correspondence: wanxa@mail.tsinghua.edu.cn

'Department of Psychology, School of Social Sciences, Tsinghua University, Qinghua Yuan, Beijing 100084, China

${ }^{2}$ Crossmodal Research Laboratory, Department of Experimental Psychology, University of Oxford, South Parks Road, OX1 3UD, Oxford, UK

Full list of author information is available at the end of the article
}

Levitan, and Spence [13]. These researchers conducted a study in which groups of participants from the United Kingdom (UK) and Taiwan viewed coloured and clear drinks and were asked what flavour first came to mind on seeing each drink. The two groups of participants exhibited different colour-flavour associations for the yellow, blue, orange, and brown drinks. For example, the most common flavour associated with the brown drink was cola for the British participants but grape for those from Taiwan. Recently, significant cross-cultural differences have also been reported in the case of colour-odour associations [14].

To date, however, most researchers have tended to argue that colour has a specific meaning regardless of the particular context in which that colour happens to have been presented. However, it would seem more likely that the same colour can have a variety of different meanings depending upon the particular context and country (or culture) in which it happens to have been presented $[15,16]$. Here, we hypothesized that one such contextual variable that might influence the meaning of 
the colour of a beverage is the container in which it happens to be presented. A dark purple coloured drink might, for example, make one think of wine if presented in a wine glass, and of grape juice, blackcurrant, or something else entirely if presented in a high ball glass.

A number of studies have already examined how the shape, size, and material of the container can influence the perception and evaluation by consumers of wine, tea, soft drinks, and water (for a recent review, see Spence et al. [17]). The shape of a glass can also influence the perceived aroma and odour of wine, at least when people are aware of the dimensions of the glass from which they happen to be tasting [18] (also see [19], for a review). The material from which a container is made has also been shown to affect participants' experience of drinking tea and soft drinks [20]. To date, however, few studies have attempted to assess how contextual variables such as the type of receptacle and culture interact to influence the specific flavours that people may associate with particular colours. The present study was therefore designed to investigate whether the type of receptacle, specifically different glasses containing a transparent coloured liquid, would influence people's colour-flavour associations. Another question of interest was whether there would be any cross-cultural differences either in terms of the crossmodal colour-flavour associations held by participants from different parts of the world, and/or in terms of the influence of the type of receptacle on the meaning of colour in those different regions.

Given that some cultural differences in colour-flavour associations have been reported previously between those participants from the UK and Taiwan [13], it seemed sensible to extend this study to investigate cross-cultural associations with participants from the United States of America (USA) and from mainland China. In this study, we asked our participants to choose the flavour (from a list) to indicate the flavour that first came to their mind when they saw pictures of drinks in different colours (red, green, yellow, blue, orange, brown, and clear) presented in four different types of receptacles, including the water glass, wine glass, cocktail glass, and plastic cup (see Figure 1 for an illustration). Specifically, we addressed the following

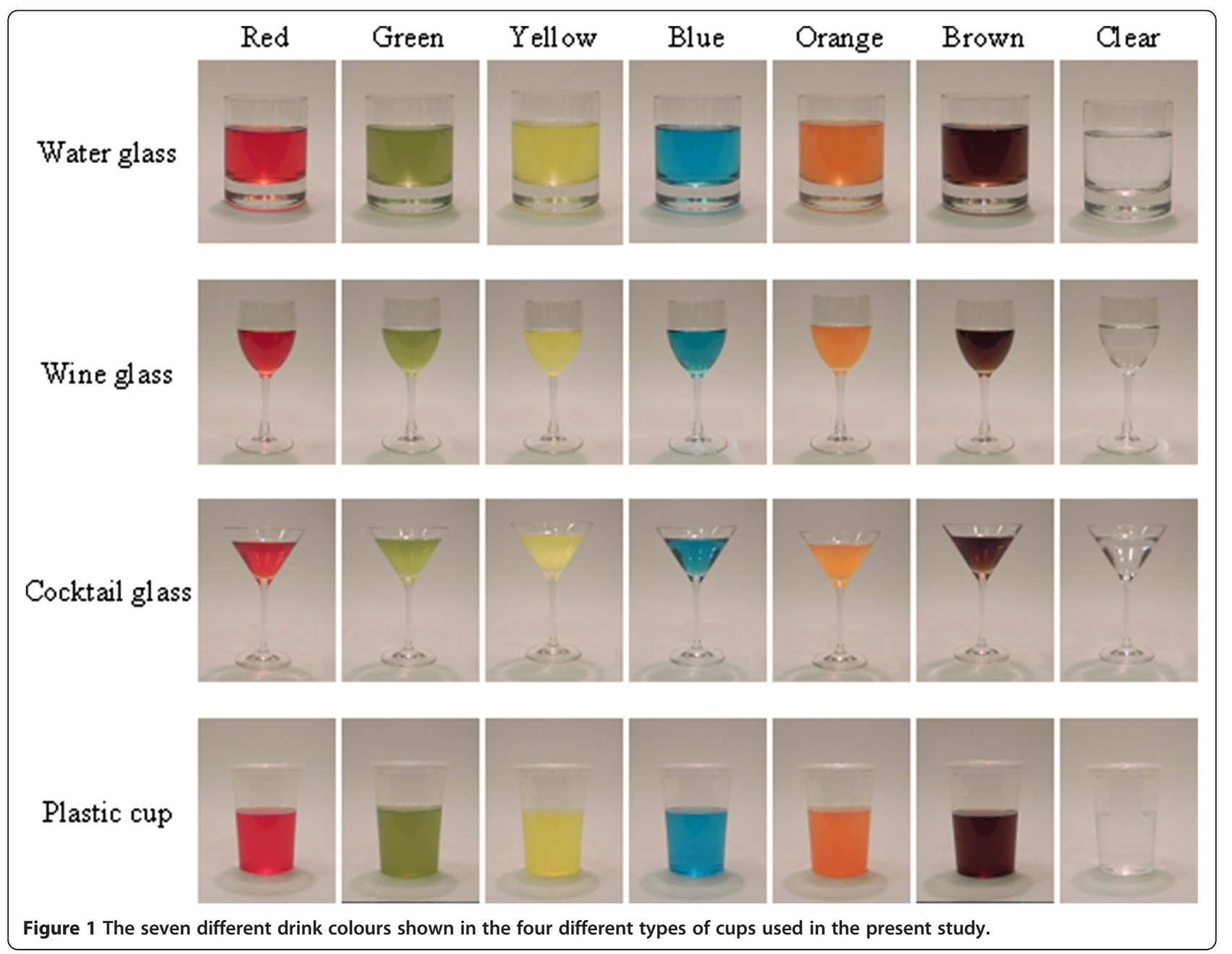


questions: First, are there any differences in the nature of colour-flavour associations between the participants from these two countries? Second, are such colour-flavour associations affected by the type of receptacle in which the coloured liquid happens to be presented? Third, how, if at all, do these two factors (the type of receptacle and culture) interact? Note also that a substantially larger sample size $(\mathrm{N}=200)$ was tested in the present study compared to that in Shankar et al.'s [13] previous study $(\mathrm{N}=35)$.

\section{Results}

The colour-flavour responses for each group of participants are highlighted in Table 1 . Note that the analysis reported here is based on that used by Shankar et al. [13]. One-sample Kolmogorov-Smirnov tests revealed that the pattern of flavours associated with each coloured drink in each type of receptacle was significantly different from a uniform distribution in both groups of participants, for all $P<.001$, suggesting the existence of robust crossmodal, colour-flavour associations in each group. Fisher's Exact Tests with $P$ values estimated with 100,000 Monte Carlo simulations and Bonferroni's correction for multiple comparisons (see [21] for the rationale for this correction) revealed that the two groups had different colour-flavour associations for (1) red or blue drinks in all type of receptacles; (2) green drinks in the water and wine glasses; and (3) yellow, orange, or brown drinks in the plastic cup, for all $P<.01$. No cross-cultural differences were observed in terms of the flavour associations for (1) the green drink presented in a cocktail glass or plastic cup; (2) the yellow,

Table 1 Top three flavour responses for each coloured drink in each type of cup in the participants from the United States of America (USA) and China (exact count in brackets)

\begin{tabular}{|c|c|c|c|c|}
\hline Colour & Cup & Participants from the USA $(\mathrm{N}=100)$ & Participants from China $(\mathrm{N}=100)$ & Difference \\
\hline \multirow[t]{4}{*}{ Red } & Water & Cherry (27), Cranberry (25), Strawberry (19) & Strawberry (27), Watermelon (25), Cherry (12) & $* *$ \\
\hline & Wine & Strawberry (28), Cherry (25), Cranberry (16) & Watermelon (24), Cherry (20), Strawberry (18) & $* *$ \\
\hline & Cocktail & Cherry (31), Strawberry (25), Cranberry (17) & Watermelon (27), Cherry (18), Strawberry (17) & ** \\
\hline & Plastic & Cherry (31), Strawberry (25), Watermelon (14) & Watermelon (35), Strawberry (20), Cherry (19) & ** \\
\hline \multirow[t]{4}{*}{ Green } & Water & Kiwi (34), Lime (27), Apple (13) & Kiwi (54), Apple (14), Lime (12) & $* *$ \\
\hline & Wine & Kiwi (31), Lime (30), Melon (12) & Kiwi (40), Apple (20), Lime (16) & ** \\
\hline & Cocktail & Lime (32), Kiwi (27), Apple (19) & Kiwi (33), Lime (23), Apple (17) & \\
\hline & Plastic & Kiwi (40), Lime (23), Apple (13) & Kiwi (58), Lime (16), Apple (7) & \\
\hline \multirow[t]{4}{*}{ Yellow } & Water & Lemon (32), Lime (24), Pineapple (14) & Lemon (26), Lime (14), Pineapple (13) & \\
\hline & Wine & Lemon (49), Lime (15), Pineapple (13) & Lemon (36), Pineapple (14), Lime (10) & \\
\hline & Cocktail & Lemon (45), Pineapple (22), Banana (9) & Lemon (25), Pineapple (19), Pear (10) & \\
\hline & Plastic & Lemon (49), Pineapple (18), Banana (13) & Lemon (28), Pineapple (26), Pear (11) & ** \\
\hline \multirow[t]{4}{*}{ Blue } & Water & Blueberry (63), Raspberry (16), Mint (8), Other (8) & Blueberry (43), Mint (30), Other (12) & ** \\
\hline & Wine & Blueberry (61), Raspberry (14), Mint (9) & Blueberry (35), Mint (31), Other (12) & $* *$ \\
\hline & Cocktail & Blueberry (59), Raspberry (18), Mint (8), Other (8) & Blueberry (32), Mint (30), Other (12) & $* *$ \\
\hline & Plastic & Blueberry (61), Raspberry (18), Other (9) & Blueberry (38), Mint (27), Other (12) & $* *$ \\
\hline \multirow[t]{4}{*}{ Orange } & Water & Orange (43), Peach (18), Mandarin (17) & Orange (24), Mandarin (21), Peach (11) & \\
\hline & Wine & Orange (46), Peach (21), Mandarin (17) & Orange (38), Mandarin (34), Peach (7) & \\
\hline & Cocktail & Orange (44), Peach (23), Mandarin (23) & Orange (32), Mandarin (31), Melon (10) & \\
\hline & Plastic & Orange (48), Peach (15), Mandarin (14) & Orange (38), Mandarin (21), Watermelon (13) & ** \\
\hline \multirow[t]{4}{*}{ Brown } & Water & Cola (66), Blackcurrant (12), Grape (9) & Cola (46), Blackcurrant (16), Other (14) & \\
\hline & Wine & Cola (68), Grape (13), Blackcurrant (10) & Cola (43), Blackcurrant (21), Other (10) & \\
\hline & Cocktail & Cola (63), Blackcurrant (23), Cranberry (4) & Cola (48), Blackcurrant (20), Other (10) & \\
\hline & Plastic & Cola (71), Grape (14), Blackcurrant (6) & Cola (32), Blackcurrant (27), Other (13) & $* *$ \\
\hline \multirow[t]{4}{*}{ Clear } & Water & Flavourless (91) & Flavourless (81), Other (6) & \\
\hline & Wine & Flavourless (88) & Flavourless (73), Other (10) & \\
\hline & Cocktail & Flavourless (86) & Flavourless (73), Other (12) & \\
\hline & Plastic & Flavourless (89) & Flavourless (71), Lychee (8), Other (8) & \\
\hline
\end{tabular}


orange, or brown drinks in the water, wine, or cocktail glass; or (3) the clear liquid in any of the receptacles, for all $P>.01$.

The participants' responses regarding the drink that they associated with each type of receptacle were classified into the following categories: (1) water, (2) alcoholic drink, (3) soda, (4) juice, (5) other, and (6) 'Don't know.' The top three drinks chosen by each group of participants are listed in Table 2. One-sample Kolmogorov-Smirnov tests revealed that the pattern of drinks associated with each type of receptacle was significantly different from a uniform distribution in both groups of participants, for all $P<.001$, thus suggesting that the participants associated each type of receptacle with a certain type of drink. The results of Fisher's Exact Tests with $P$ values estimated with 100,000 Monte Carlo simulations and Bonferroni's correction for multiple comparisons suggested that the participants from the USA and China had associated each type of receptacle with different drinks. As for the water glass, alcohol was the drink that most often came to mind for the participants from the USA, whereas the Chinese participants thought of water instead. As for the wine and cocktail glasses, although alcohol was most often associated for both groups, more of the participants from the USA than from China (90\% versus $60 \%$, respectively) provided alcohol as their answer. As for the plastic cup, although water was the most frequent answer for both groups, the second most frequently chosen answer was soda for the participants from the USA, and 'Don't know' for the participants from China.

\section{Discussion}

\section{Cross-cultural differences in receptacle-colour-flavour} associations

Both the statistics and counts of chosen flavours consistently demonstrated the interaction between the type of

Table 2 Top three drinks associated with each type of cup/glass for the groups of participants from the United States of America (USA) and from China when containing clear water (exact count in brackets)

\begin{tabular}{|c|c|c|c|}
\hline Cup & $\begin{array}{l}\text { Participants from the } \\
\text { USA }(N=100)\end{array}$ & $\begin{array}{l}\text { Participants from } \\
\text { China }(N=100)\end{array}$ & Difference \\
\hline Water glass & $\begin{array}{l}\text { Alcohol (49), } \\
\text { Water (39) }\end{array}$ & $\begin{array}{l}\text { Water (52), } \\
\text { Alcohol (16), } \\
\text { Juice (10) }\end{array}$ & ** \\
\hline Wine glass & $\begin{array}{l}\text { Alcohol (90), } \\
\text { Water (8) }\end{array}$ & $\begin{array}{l}\text { Alcohol (60), } \\
\text { Soda (9), } \\
\text { Juice (9) }\end{array}$ & $* *$ \\
\hline Cocktail glass & Alcohol (93) & $\begin{array}{l}\text { Alcohol (58), } \\
\text { Don't know (17), } \\
\text { Water (9), Juice (9) }\end{array}$ & $* *$ \\
\hline Plastic cup & $\begin{array}{l}\text { Water (58), } \\
\text { Soda (22) }\end{array}$ & $\begin{array}{l}\text { Water (48), } \\
\text { Don't know (18), } \\
\text { Soda (12) }\end{array}$ & $* *$ \\
\hline
\end{tabular}

Note: ${ }^{* *} P<.01$ receptacle and the cultural background of the participants on the flavour associations elicited by the green, yellow, orange, and brown drinks. That is, the crosscultural difference for yellow, orange, and brown drinks emerged only when the drinks were presented in the plastic cup. This result allowed us to compare these results directly to those reported by Shankar et al. [13]. In the latter study, all of the drinks were presented in plastic cups. Shankar and colleagues also found crosscultural differences in terms of the particular colour-flavour associations for yellow, orange, and brown drinks. Our results confirmed the cross-cultural difference in the associations between flavour expectations and the yellow, orange, and brown beverage colours. In addition, our results also highlight the possibility that such cross-cultural differences are very likely to occur when a drink is presented in a plastic cup.

As for the green drink presented in a plastic cup, the most common flavour choice was kiwi for all participants. In contrast, Shankar et al. [13] reported that participants from the UK and Taiwan consistently chose the flavour of mint for a green drink when it was presented in a plastic cup, whereas kiwi was mentioned less often. These results might be taken to suggest another crosscultural difference, but they might also be attributed to the fact that the green drinks presented in these two studies were not exactly the same.

Taken together, the findings of the present study suggest how the type of receptacle, as a contextual factor, can interact with a participant's cultural background in order to influence the flavour expectations they hold for green-, yellow-, orange-, and brown-coloured drinks. According to the associative learning account of crossmodal correspondences, people are sensitive to the repeated exposure to specific combinations of stimuli [22]. What is more, consumers presumably learn crossmodal associations between colours and flavours across the lifespan as a result of their being exposed to specific combinations of stimuli (colours and flavours) that happen to co-occur together. Those combinations may differ across cultures, thus leading different groups of consumers to hold different crossmodal colour-flavour associations. Furthermore, the cross-cultural differences in colour-flavour associations are also presumably based on different expectations generated from the repeated co-occurrence of certain pairings of colour and flavour in everyday life. These cross-cultural differences may also result from a participant's exposure to different commercial products and to differences in agriculture practices in different environments [13]. The influence of the type of receptacle on such cross-cultural differences suggests that participants' expectations might be strengthened, weakened, or otherwise altered by contextual factors, such as the type of container in which a drink is shown (e.g., as in an advertisement). 


\section{Cross-cultural differences in colour-flavour associations}

The present results revealed a cross-cultural difference in the associations between flavour expectations that were elicited by the sight of a red beverage, which was not influenced by the type of receptacle in which the beverage was presented. For those participants from the USA, cherry flavour was most commonly associated with the red drink in three out of four containers, whereas the most frequent flavour choice for the same drink for the Chinese participants was watermelon. This crosscultural difference might well be attributed to the fact that the same colour red is repeatedly seen with different flavours in the marketplace of these two countries. Although red is associated with love and happiness in both countries [23], it is reported as being associated with 'good-tasting' in the USA, but not by those from China. It is therefore difficult to determine whether documented cross-cultural differences in the colour-flavour associations arise at a perceptual or at a more semantic level [10,24].

Similarly, the cross-cultural difference in the crossmodal associations between flavour and the blue-coloured beverage was not influenced by the type of receptacle either. In the present study, the cross-cultural difference was shown for the second most common flavour choice. Blueberry was the flavour that was most often associated with the blue-coloured drinks by both groups of participants. The next most common choice was raspberry for the participants from the USA but mint for those from China. This difference might perhaps be attributable to the fact that raspberry is not a particularly common fruit in China. Interestingly, blue was rated as the most liked colour by participants from eight different countries, including the USA and China [15], and they both associate it with high quality [23].

\section{The impact of the container on drink expectations}

Interestingly, the results of the present study demonstrate that participants from the USA and China associated the same container with different drinks. Alcohol most often came to the minds of the USA participants' when viewing the water glass, whilst it was water that came to mind for Chinese participants. As for the wine and cocktail glasses, although alcohol was the drink that was most often associated for both groups, more of the participants from the USA than from China suggested alcohol. Therefore, it would seem reasonable to expect that these two groups of consumers might have different expectations regarding the flavour of one and the same coloured drink if shown in an online advertisement, say. For example, when presented in a water glass, an alcoholic drink might be judged as highly 'appropriate' for those from the USA, while not eliciting such a strong feeling in those from China. Given that expectations concerning the flavour of a drink have been shown to influence people's subjective evaluation of the drink [25], the present findings provide evidence in support of the idea that it is important to choose the type of receptacle in which to present coloured drinks carefully, based on both the colour of the drink and the culture background of the audience.

\section{Limitation and future directions}

Further research will be needed in order to overcome the limitations of the present study. First, it should be noted that the two groups of participants tested here differed not only in terms of their culture but also in terms of their age, as those participants who were recruited from the USA were older than those who took part in the study from China. It is possible that their age might also have influenced their familiarity with certain alcoholic drinks or the expectations on the drinks presented to them, but future research is needed to test this possibility. Second, when the participants were asked to indicate the first flavour that came to mind for each drink, they were asked to choose just one flavour from a list. Although this method has commonly been used in the literature [10], it is important to note that it may not accurately represent the colour-flavour associations that a participant might come up with spontaneously (that is, it fails to capture the strength of the association for a given individual). However, that said, it is worth noting that Shankar et al. [13] had their participants freely write down any flavour that they thought of when seeing each coloured drink, and many of the results we obtained with providing a list to choose from were consistent with those freely written down by their participants. Third, the participants from both the U.S.A. and China finished the experiment on their own computers and saw the photos of coloured drinks on their own monitors, so we had no control over the colours that they actually saw. This is, of course, also exactly the same challenge that faces any international marketer wanting to show their latest product offerings online.

\section{Conclusions}

In summary, the results of the present study suggest that the type of the container influences the colour-flavour associations for certain beverage colours, and also reveals some cross-cultural differences between participants from the USA and China in terms of their colourflavour associations. The two groups of participants exhibited different colour-flavour associations for the green, yellow, orange, and brown drinks when presented in the different receptacles, whereas the cross-cultural differences in the colour-flavour associations for red and blue drinks were independent of the type of container used to present the drinks. These findings therefore highlight an interaction between contextual factors and 
culture in terms of colour-flavour associations. These findings have relevance to the international marketing of drinks. It is worth remembering that many international drinks manufacturers are currently thinking about how best to break into the Chinese market, which undoubtedly represents a huge area of potential growth for many businesses. We predict that online marketing, possibly using images and presentation formats similar to those utilized here (for example, online testing) is going to become increasingly popular and important in the years to come. Critically, previous research has tended to assess the meaning of different colours for flavour perception without necessarily taking into account (or at the very least without giving sufficient consideration to) the receptacle in which the coloured liquid happens to have been presented. The results reported here demonstrate, for what we believe to be the first time, that a given beverage colour can have a very different meaning (or associated flavour) depending on the receptacle in which it is presented - which can be thought of as a kind of context effect [17] - as well as the country in which it is presented. We would argue though that the findings of the present study should be intriguing enough to capture the attention of those marketers who wish to better tailor their products by using appropriate colour-flavour pairs for the consumers from different countries and by using the most appropriate/flattering receptacle.

\section{Methods}

\section{Participants}

One hundred participants from mainland China (50 women and 50 men, mean age $=27.3$ years, $\mathrm{SD}=1.8$, ranging from 20 to 32 years) were recruited to take part in this study from the subject pool of the Spatial Cognition Lab at the Psychology Department of Tsinghua University; 100 participants from the USA (44 women and 56 men, mean age $=32.9$ years, $\mathrm{SD}=11.2$, ranging from 19 to 61 years) were recruited from Amazon's Mechanical Turk in exchange for a payment of 0.80 US dollars. All of the participants provided informed consent prior to their taking part in the study. The experiment was approved by the Central University Research Ethics Committee of the University of Oxford.

\section{Apparatus and materials}

Photos of coloured-water drinks (180 pixels wide $\times 240$ pixels high) were shown to participants. The drinks were made using $400 \mathrm{ml}$ plastic cups (Tesco, Cheshunt, UK) to mix $250 \mathrm{ml}$ of water with the different commercial food colourings (Dr. Oetker, Leeds, UK) to achieve six colours: red $(.30 \mathrm{ml}$ red food colouring), green $(.45 \mathrm{ml}$ green and .05 yellow food colouring), yellow $(.05 \mathrm{ml}$ yellow food colouring), blue (.20 blue food colouring), brown $(.15 \mathrm{ml}$ red, $.10 \mathrm{ml}$ blue, and $.10 \mathrm{ml}$ yellow food colouring), and orange $(.10 \mathrm{ml}$ yellow and $.05 \mathrm{ml}$ red food colouring). Clear water was also presented as the seventh condition. As shown in Figure 1, the drinks were presented in four different containers, including a water glass, a wine glass, a cocktail glass, and a plastic cup.

Both groups of participants took part in the study online using their own computer on two websites. The participants from China completed the task in Chinese via Unipark (www.unipark.de), whereas the participants from the USA completed it in English via Xperiment (http:// www.xperiment.mobi downloaded on 15 May 13).

\section{Design and procedure}

A 2 (Culture: USA versus China) $\times 4$ (Receptacle: water glass, wine glass, cocktail glass, or plastic cup) design was used, with Culture as a between-group factor and Receptacle as a within-group factor. Each participant completed 28 trials (7 colours $\times 4$ types of receptacle) presented in a random order.

Before starting the main experiment, the participants were informed about the general aims of the study, and only those participants who accepted to take part continued. During each trial, a photo of a drink was shown, and the participant had to choose the first flavour or drink that came to mind based on the drink's colour from a list of 24 flavours, including apple, banana, blackcurrant, blueberry, cherry, cola, cranberry, grape, kiwi, lemon, lime, lychee, mandarin, melon, mint, orange, peach, pear, pineapple, raspberry, strawberry, watermelon, flavourless, and other. The list was constructed by combining those flavour options found in the papers of Shankar et al. [13] and Zampini et al. [10], the wine aroma wheel (winearomawheel.com), and several flavours that are commonly found in the Chinese marketplace. These options were presented in a random order in each trial to avoid any possible order or position effects. The participants were instructed to specify which flavour they had in mind if they chose the 'other' option instead ${ }^{\mathrm{a}}$. At the end of the experiment, the participants were shown the pictures of four receptacles with clear water in them (in a random order) and asked to specify the drink they associated with the receptacle or answer 'Don't know' if they were unable to provide an answer. The experiment lasted approximately 15 minutes.

\section{Endnotes}

${ }^{\mathrm{a}}$ After participants were asked to choose the first flavour they thought of when seeing the photo of a coloured drink, and they were also asked to rate the drink for familiarity and how pleasant they expected it to be using a 7-point Likert scale. The data are not reported here because they fall outside the scope of this paper. 


\section{Competing interests}

The authors declare that they have no competing interests.

\section{Authors' contributions}

Each of the listed co-authors made the following contributions to the paper: XW, CS, and CV co-developed the idea for the study and collaboratively designed the study. CM and XW designed the stimuli for the study. BM and AT collected the data and revised the manuscript. XW and CS conducted the data analysis and interpretation of the data, and drafted the manuscript. CV, AT, and BM aided in the initial stages of the manuscript's preparation. All of the authors have read and approved the final version of the manuscript.

\section{Acknowledgements}

This research was supported by Tsinghua University Cultural Inheritance and Innovation Program and by the Tsinghua-Santander Young Faculty Program. The authors would like to thank Xi Zhou for her assistance in data collection. Comments concerning this paper should be sent to Dr. Xiaoang Wan at wanxa@mail.tsinghua.edu.cn.

\section{Author details}

${ }^{1}$ Department of Psychology, School of Social Sciences, Tsinghua University, Qinghua Yuan, Beijing 100084, China. ${ }^{2}$ Crossmodal Research Laboratory, Department of Experimental Psychology, University of Oxford, South Parks Road, OX1 3UD, Oxford, UK. ${ }^{3}$ Xperiment, Lausanne, Switzerland.

Received: 28 November 2013 Accepted: 29 January 2014

Published: 25 February 2014

\section{References}

1. Auvray M, Spence C: The multisensory perception of flavor. Conscious Cogn 2008, 17:1016-1031.

2. Zellner DA: Color-odor interactions: a review and model. Chem Percept 2013, 6:155-169.

3. DuBose CN, Cardello AV, Maller O: Effects of colorants and flavorants on identification, perceived flavor intensity, and hedonic quality of fruit-flavored beverages and cake. J Food Sci 1980, 45:1393-1399.

4. Hyman A: The influence of color on the taste perception of carbonated water preparations. B Psychonomic Soc 1983, 21:145-148.

5. Morrot G, Brochet F, Dubourdieu D: The color of odors. Brain Lang 2001, 79:309-320.

6. Shankar M, Simons C, Shiv B, Levitan C, McClure S, Spence C: An expectations-based approach to explaining the influence of color on odor identification: the influence of degree of discrepancy. Atten Percept Psychophys 2010, 72:1981-1993.

7. Stillman JA: Color influences flavor identification in fruit-flavored beverages. J Food Sci 1993, 58:810-812.

8. Wood AT, Lloyd DM, Kuenzel J, Poliakoff E, Dijksterhuis GB, Thomas A: Expected taste intensity affects response to sweet drinks in primary taste cortex. Neuroreport 2011, 22:365-369.

9. Wood AT, Poliakoff E, Lloyd DM, Dijksterhuis GB, Thomas A: Flavour expectation: the effect of assuming homogeneity on drinking perception. Chem Percept 2010, 3:174-181.

10. Zampini M, Sanabria D, Phillips N, Spence C: The multisensory perception of flavor: assessing the influence of color cues on flavor discrimination responses. Food Qual Prefer 2007, 18:975-984.

11. Zellner DA, Bartoli AM, Eckard R: Influence of color on odor identification and liking ratings. Am J Psychol 1991, 104:547-561.

12. Aslam MM: Are you selling the right colour? A cross-cultural review of colour as a marketing cue. J Marketing Commun 2006, 12:15-30.

13. Shankar MU, Levitan C, Spence C: Grape expectations: the role of cognitive influences in color-flavor interactions. Conscious Cogn 2010, 19:380-390

14. Ren J, Woods A, McKenzie K, Ru LX, Levitan CA: Cross-cultural colour-odour associations. Appetite 2012, 59:634.

15. Madden TJ, Hewett K, Roth MS: Managing images in different cultures: a cross-national study of color meanings and preferences. J Int Marketing 2000, 84:90-107.

16. Piqueras-Fiszman B, Velasco C, Spence C: Exploring implicit and explicit crossmodal colour-flavour correspondences in product packaging. Food Qual Prefer 2012, 25:148-155.
17. Spence C, Harrar V, Piqueras-Fiszman B: Assessing the impact of tableware and other contextual variables on multisensory flavour perception. Flavour 2012, 1:7.

18. Hummel T, Delwiche JF, Schmidt C, Hüttenbrink KB: Effects of the form of glass on the perception of wine flavors: a study in untrained subjects. Appetite 2003, 41:197-202.

19. Spence C: Crystal clear or gobbletigook? World Fine Wine 2011, 33:96-101.

20. Schifferstein HNJ: The drinking experience: cup or content? Food Qual Prefer 2009, 20:268-276.

21. Maxwell SE, Delaney HD: Designing experiments and analyzing data: a model comparison perspective. Mahwah NJ: Erlbaum; 2004.

22. Wilson DA, Stevenson RJ: Learning To Smell: Olfactory Perception From Neurobiology To Behavior. Baltimore, MD: The Johns Hopkins University Press; 2006.

23. Jacobs L, Keown C, Worthley R, Ghymn Kl: Cross-cultural colour comparisons: global marketers beware! Int Marketing Rev 1991, 83:21-31.

24. Spence C: Crossmodal correspondences: a tutorial review. Atten Percept Psychophys 2011, 73:971-995.

25. Raudenbush B, Meyer B, Eppich W, Corley N, Petterson S: Ratings of pleasantness and intensity for beverages served in containers congruent and incongruent with expectancy. Percept Motor Skill 2002, 94:671-674.

doi:10.1186/2044-7248-3-3

Cite this article as: Wan et al.: Does the type of receptacle influence the crossmodal association between colour and flavour? A cross-cultural comparison. Flavour 2014 3:3.

\section{Submit your next manuscript to BioMed Central and take full advantage of:}

- Convenient online submission

- Thorough peer review

- No space constraints or color figure charges

- Immediate publication on acceptance

- Inclusion in PubMed, CAS, Scopus and Google Scholar

- Research which is freely available for redistribution 